Document downloaded from:

http://hdl.handle.net/10251/69126

This paper must be cited as:

Ramirez Hoyos, P.; Gómez Lozano, V.; Cervera, J.; Nasir, S.; Ali, M.; Ensinger, W.; Mafe, S. (2015). Energy conversion from external fluctuating signals based on asymmetric nanopores. Nano Energy. 16:375-382. doi:10.1016/j.nanoen.2015.07.013.

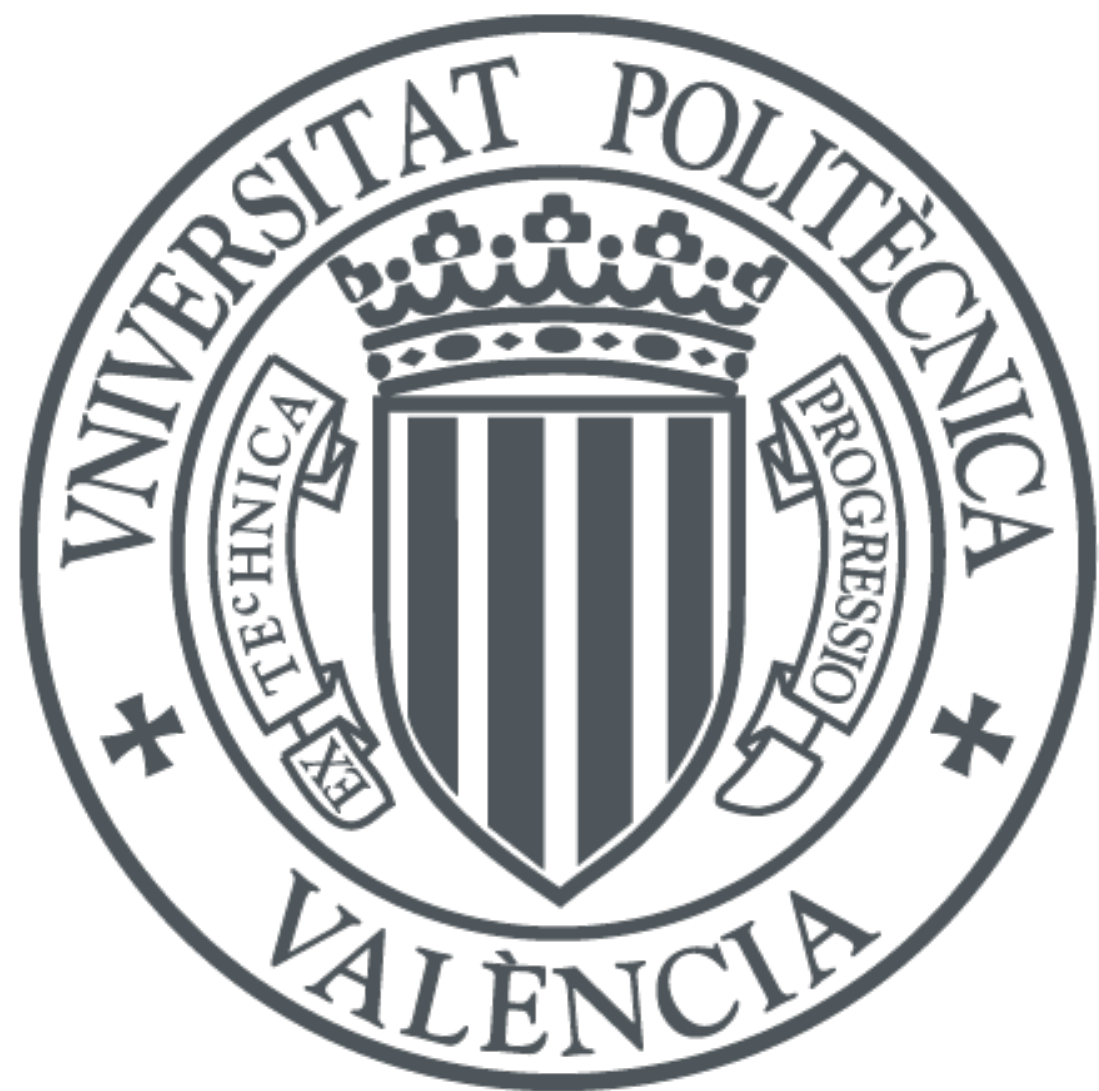

The final publication is available at

http://dx.doi.org/10.1016/j.nanoen.2015.07.013

Copyright Elsevier

Additional Information 


\section{Energy conversion from external fluctuating signals based on asymmetric nanopores}

Patricio Ramirez, ${ }^{1,}$ Vicente Gomez, ${ }^{1}$ Javier Cervera, ${ }^{2}$ Saima Nasir, ${ }^{3,4}$ Mubarak Ali, $^{3,4}$ Wolfgang Ensinger ${ }^{3,4}$ and Salvador Mafe ${ }^{2}$

${ }^{1}$ Dept. de Física Aplicada, Universitat Politècnica de València, E-46022 València, Spain.

${ }^{2}$ Dept. de Física de la Tierra i Termodinàmica, Universitat de València, E-46100 Burjassot, Spain.

${ }^{3}$ Dept. of Material- and Geo-Sciences, Materials Analysis, Technische Universität Darmstadt, D-64287 Darmstadt, Germany

${ }^{4}$ Materials Research Department, GSI Helmholtzzentrum für Schwerionenforschung, D-64291, Darmstadt, Germany

*Corresponding author. Email address: patraho@fis.upv.es (P. Ramirez)

\section{Abstract}

Electrical transduction from fluctuating external signals is central to energy conversion based on nanoscale electrochemical devices and bioelectronics interfaces. We demonstrate theoretically and experimentally a significant energy transduction from white noise signals using the electrical rectification of asymmetric nanopores in polymeric membranes immersed in aqueous electrolyte solutions. Load capacitor voltages of the order of $1 \mathrm{~V}$ are obtained within times of the order of 1 min by means of nanofluidic diodes which convert zero time-average potentials of amplitudes of the order of $1 \mathrm{~V}$ into average net currents. We consider singlenanopore and multipore membranes to show that the conversion processes can be significantly increased by scaling. The results concern a wide range of operating electrolyte concentrations. Because these concentrations dictate the pore resistance, the tuning of the load capacitance to optimize the system response at each concentration is also addressed. Finally, the experimental results are described theoretically by using simple equivalent circuits with a voltage-dependent resistance in series with a load capacitance.

KEYWORDS: nanopore, fluctuating signal, electrical rectification, energy conversion 


\section{Introduction}

The electrical transduction of fluctuating signals is central to energy conversion based on nanoscale electrochemical devices, being significant also for bioelectronics interfaces. We have demonstrated recently [1] that electrical rectification with nanofluidic diodes $[2,3]$ allows significant energy conversion for the case of a single nanopore and large amplitude white noise signals. To this end, external electric potentials of zero average were converted into net currents, allowing an external load capacitor to reach a voltage of the order of $1 \mathrm{~V}$ in a time of the order of $1 \mathrm{~min}$ [1]. This fact clearly suggests that soft matter nanostructures operating in an aqueous ionic solution can effectively be coupled to conventional electronic elements like commercial capacitors. Because of the biomimetic nature of the pore, the results have clear implications for sensing and information processing using bioelectronic interfaces and nanopore-based biosensors [4-11]. We note in this context that the artificial nanopore used here shows electrical rectification and ionic selectivity characteristics similar to those observed in wide ion channels (e.g., the bacterial porin $O m p F$ of Escherichia coli, a protein pore reconstituted on a planar lipid bilayer $[12,13])$.

We present here a significant extension of the above results concerning these biomimetic nanomaterials $[1,12]$, paying attention to the energy conversion processes. First, we use both single-nanopore and multipore membranes in an effort to demonstrate that the conversion processes are robust and can be enhanced significantly by simple scaling. Second, we consider a wide range of electrolyte concentrations to better simulate real operating conditions. Third, we address the tuning of the load capacitance at each concentration (note that this concentration dictates the nanopore resistance). Finally, we show that the observed phenomena can be analyzed theoretically by using simple equivalent circuits with a voltage-dependent resistance in series with a load capacitance. This question should be significant to simulate the expected system response in each practical case. 


\section{Experiment and numerical methods}

Nanopore. Polymer foils of polyethylene terephthalate (PET) of thickness $12 \mu \mathrm{m}$ (Hostaphan RN 12, Hoechst) were irradiated either with single or $10^{4}$ swift heavy ions $\mathrm{cm}^{-2}(\mathrm{~Pb}, \mathrm{U}$, and $\mathrm{Au})$, with an energy of 11.4 MeV per nucleon, at the UNILAC linear accelerator (GSI, Darmstadt). A single conical pore was obtained by asymmetric track-etching methods [14,15], which give typical radii in the range $10-20 \mathrm{~nm}$ for the cone tip and $100-200 \mathrm{~nm}$ for the cone basis. The approximate pore radii can be obtained from the steady-state current $(I)$ - potential $\left(V_{N}\right)$ curve in each case, with $V_{N}=V_{R}-V_{C}$ (Fig. 1a and b) $[14,15]$. Three single pore samples were used to clearly show the electrical characteristics of the nanostructures. An additional sample was also prepared with a multipore membrane etched together with the single-pore membrane. The etching process results in carboxylic acid groups located on the pore surface. These groups are ionized in a $0.1 \mathrm{M} \mathrm{KCl}$ aqueous solution at $p H=7$. Each nanopore has then a negative charge distribution along the axis, which is asymmetric because of the approximately conical pore geometry [16]. This charge asymmetry is responsible for the electrical rectification of the $I-V_{N}$ curve (Fig. 1b and c), which permits the transduction of white noise signal (Fig. 1d). Control experiments were also conducted at low $p H(p H=3$, neutral pore [1]). Before and after each measurement, the $p H$ was checked by using a Crison GLP22 $\mathrm{pH}$-meter.

Measurements. A couple of $\mathrm{Ag} \mid \mathrm{AgCl}$ electrodes immersed in the bathing solutions (Fig. 1a) allowed the fluctuating electric potentials to be applied. Note that the current entering the (highly charged) cone tip opening of the pore finds an electrical resistance lower than that entering the wide basis opening, which gives the observed electrical rectification. The current-voltage curves were measured with one picoammeter/voltage source (Keithley 6487/E). The voltages across the capacitor of Fig. 1a were measured with a multimeter (Keithley 2000/E). The recorded currents $I(t)$ allowed to obtain the average currents $<I>$ of Fig. 2 a. 
Simulations. The nanopore is considered as a potential dependent resistance connected in series to the capacitor (Fig. 1a). To simulate the charging process, the electrical circuit was fed with fluctuating potential signals of amplitude $V_{0}$. The charging process was described by the equation $\mathrm{d} V_{C}(t) / \mathrm{d} t=I(t) / C$, where $V_{C}(t)$ and $I(t)$ are the capacitor potential and the current, respectively. The initial condition was $V_{C}(t=0)=0$ and the constant time step $\Delta t=0.05 \mathrm{~s}$, which is of the same order of magnitude as the steps in the experiments. $V_{R}(t)$ took random values between $-V_{0}$ and $+V_{0}$. The capacitor voltage was updated as $V_{C} \rightarrow V_{C}+I\left(V_{N}\right) \Delta t / C$ at each time step $\Delta t$, where $I\left(V_{N}\right)$ is the experimental current of Fig. 1b. The charging process eventually gave a maximum voltage $V_{C}(\infty)$. To describe the discharging process, we assumed the condition $V_{R}(t)=0$ in the simulations. The simulations were performed using a Python numerical code.

\section{Results and Discussion}

Fig. 1a shows the experimental set-up. A commercial capacitor of capacitance $C$ is connected in series to either the single conical nanopore or the multipore membrane. The pore is immersed in two identical $\mathrm{KCl}$ aqueous solutions of concentration $c_{0}$. Fig. $1 \mathrm{~b}$ shows the steady-state current $(I)$ - voltage $\left(V_{N}\right)$ curves obtained for three single-pore membranes (samples 1-3) with different rectification characteristics (Fig. 1c). The results correspond to $c_{0}=0.1 \mathrm{M}$ and $p H=7$, which gives an asymmetrically distributed, negative charge along the axis of the conical pore. This charge is due to the ionized carboxylate $\left(\mathrm{COO}^{-}\right)$groups on the pore surface, which explains the rectification observed in the $I-V_{N}$ curves [2,15-17].

When a white noise, randomly fluctuating electric potential $V_{R}(t)$ of amplitude $V_{0}$ is externally applied in the $\mathrm{Ag} \mid \mathrm{AgCl}$ electrodes (the input signal of Fig. 1d), a time $(t)$-dependent electric current $I(t)$ with a non-zero time average $\langle I>$ is obtained (the output signal of Fig. 1d). 
This net current, which corresponds to sample 3 here, allows charging the load capacitor of Fig. 1a. Control experiments at low $p H$ (not shown here) give zero net currents, $\langle I>=0$, because of the ohmic pore behavior (no rectification) [1]. These results confirm that $\langle I>$ arises from the rectification of the external fluctuating signal by the asymmetric nanostructure and is not disturbed significantly by other internal noise sources of the electrical equipment [1]. Note also that the output electric current $I(t)$ is slave of the input potential $V_{R}(t)$ because the period of this potential is much longer than the relaxation time characteristic of the small solution volumes found in nanopores [17-19] and ion channels [12]. This (adiabatic) limit is valid for low enough frequency signals $[1,12,18-21]$.

Fig. 2a and $\mathrm{b}$ show the average currents $\langle I>$ and electrical resistances $R$ obtained for the single-pore samples 1-3, respectively. High average currents leading to significant capacitor charging are obtained for low pore resistances (Fig. 2b) and high rectifications (Fig. 1c). Fig. 2c and $\mathrm{d}$ show the charging and discharging curves for the three samples of Fig. 2a. In these experiments, the voltage amplitude is $V_{0}=1 \mathrm{~V}$, the load capacitance is $C=1 \mu \mathrm{F}$, and the electrolyte concentration is $c_{0}=0.1 \mathrm{M} \mathrm{KCl}$. The saturation capacitor voltage $V_{C}(t \rightarrow \infty) \equiv V_{C}(\infty)$ obtained at long times (Fig. 2c) is determined by the rectification ratio of Fig. 1c (compare this figure with Fig. 2a for $<I>$ ).

Remarkably, Fig. 2c shows that fluctuating potentials with amplitudes of the order of $1 \mathrm{~V}$ result in capacitor voltages of the order of $1 \mathrm{~V}$ within a few minutes. Note also that Fig. 2c shows the same time pattern in all charging curves because we use the same scheme for the input potential pulses (scaling the single point values to the fixed amplitude). This procedure would permit to identify other internal noise sources (wiring, capacitor, and voltage sources) in case they were present. The capacitor discharging follows an approximately exponential decay when the fluctuating external potential is disconnected (Fig. 2d). The resistance of the single pore is lower than $0.1 \mathrm{G} \Omega$ for most of the cases in Fig. 2b. Because the load capacitor of Fig. 1a has a 
capacitance $C=1 \mu \mathrm{F}$ in these experiments, an equivalent $R C$ circuit time is of the order of $10-$ $100 \mathrm{~s}$, which is in agreement with the experimental curves of Fig. 2c and d. In particular, the experimental charging times obtained are between $22 \mathrm{~s}$ (sample 3) and $82 \mathrm{~s}$ (sample 2).

Alternatively, the above energy conversion process can be enhanced by orders of magnitude using a multipore membrane (Fig. 3) instead of the single pore of Fig. 1 and Fig. 2. Indeed, Fig. 3a shows that steady state currents of the order of $0.1 \mathrm{~mA}$ can be obtained with a nanoporous membrane of approximately $10^{4}$ conical pores $/ \mathrm{cm}^{2}$ at different electrolyte concentrations (the measured currents were of the order of $10 \mathrm{nA}$ only in Fig. 1b). Also, the high capacitance $C=10 \mathrm{mF}$ used in Fig. 3d allows collecting approximately 10000 times the charge stored with the single pore (note that $C=1 \mu \mathrm{F}$ in Fig. 2c) for the same capacitor voltage. Although the energy conversion can be enhanced further by simple scaling, this enhancement cannot be maintained indefinitely. It has been experimentally demonstrated [22] that membrane samples with high pore densities $\left(\approx 10^{6}\right.$ conical pores $\left./ \mathrm{cm}^{2}\right)$ obtained by track-etching display very low rectification ratios. This is due to the overlapping of adjacent pores that merge into each other, giving place to wide pores where the fixed charge effects are screened by the mobile ions. As a result, the membrane sample shows an almost linear current-voltage curve that would lead in our case to negligible capacitor charging [1].

The effect of the electrolyte concentration $c_{0}$ on the steady-state $I-V_{N}$ curve and electrical rectification of a multipore membrane is also shown in Fig. $3 \mathrm{a}$ and $\mathrm{b}$. The increase of $c_{0}$ leads to an increase in the number of electrical carriers (ions here), and then to high currents (Fig. 3a). However, this effect gives low rectification ratios for high enough values of $c_{0}$ (Fig. $3 \mathrm{~b}$; a maximum rectification is achieved at $c_{0}=0.05-0.1 \mathrm{M}$, as previously reported for single pore membranes [16]). It is well-known that the nanopore selectivity and rectification characteristics can be optimized at intermediate electrolyte concentrations $[16,17]$ because these concentrations 
are high enough for the absolute currents to be significant but low enough for electrical rectification to be significant [16].

Also, Fig. 3c shows that the membrane electrical resistance $R$ decreases by a factor 10 when increasing the concentration $c_{0}$ from $0.01 \mathrm{M}$ to $1 \mathrm{M}$. This experimental fact significantly modifies the electrical characteristics of the equivalent circuit in Fig. 1a, making it necessary to tune the capacitance $C$ as a function of $c_{0}$ in order to obtain robust charging curves using the white noise signal (see Fig. 3d, obtained for a fixed capacitance $C=10 \mathrm{mF}$ ).

Fig. 4a shows the capacitance tuning effect for a multipore membrane and the load capacitances $C=1$ and $10 \mathrm{mF}\left(c_{0}=0.1 \mathrm{M} \mathrm{KCl}\right.$ and $V_{0}=2 \mathrm{~V}$ here). For low values of $C$, the charging curve shows high noise and then the saturation capacitor voltage $V_{C}(\infty)$ is not well defined. A high value of $C$ can solve this problem at the price of long charging and discharging times (Fig. $4 \mathrm{a}$ and $\mathrm{b}$ ). Note also that $V_{C}(\infty)$ is dictated by the electrical rectification, not by the capacitance. The theoretical simulations for the charging (Fig. 4a) and discharging (Fig. 4b) curves reproduce the experimental trends.

Figs. 5a and $\mathrm{b}$ show the charging and discharging curves obtained using the multipore membrane for different voltage amplitudes $V_{0}$, with $C=10 \mathrm{mF}$ and $c_{0}=0.1 \mathrm{M} \mathrm{KCl}$. The capacitor saturation voltages $V_{C}(\infty)$ increase with the amplitude $V_{0}$ of the input signals and are achieved when the reverse current which opposes the charging process becomes equal to the net charging current [1]. Again, we use the same time pattern scheme for the input potential pulses as in Fig. 2c, scaling the single point values to the fixed amplitude. The theoretical charging (Fig. 4a) and discharging (Fig. 4b) curves reproduce the experimental trends. Note that $V_{C}(\infty)$ depends linearly on the white noise amplitude $V_{0}$ (Fig. 5a), which is correctly described by the theoretical simulations. 
Figs. 6a shows the energy $E=C V_{C}(\infty)^{2} / 2$ stored in the load capacitor as a function of the concentration $c_{0}$ at $V_{0}=2 \mathrm{~V}$. Fig. $6 \mathrm{~b}$ shows this energy as a function of the voltage amplitude $V_{0}$ at $c_{0}=0.1 \mathrm{M}$. This energy is of the order of $1 \mathrm{~mJ}$ for a noise amplitude of $1 \mathrm{~V}$ and $C=10 \mathrm{mF}$. The maximum of $E$ achieved at intermediate values of the electrolyte concentration $c_{0}$ (Fig. 6a) is given by the maximum of $V_{C}(\infty)$, which is dictated in turn by the maximum electrical rectification observed in Fig. 3b. Note also that $E$ depends on $V_{0}^{2}$ in Fig. 6 b because of the linear dependence between $V_{C}(\infty)$ and the noise amplitude $V_{0}$ in Fig. 5a.

Finally, the energy stored in the load capacitor (Fig. 6a and b) can be compared with the input energy $E_{\text {in }}=\int_{0}^{T} I(t) V_{R}(t) \mathrm{d} t$. To obtain the latter energy, we assume an effective time $T=$ $3 \tau_{c h}$ for the limiting value of the voltage capacitor [1], where $\tau_{c h}$ is the charging time. From the experimental time traces obtained for the multipore membrane, the output energy is between $1.7 \%\left(V_{0}=0.5 \mathrm{~V}\right)$ and $11 \%\left(V_{0}=3 \mathrm{~V}\right)$ of the input energy. Additional experiments with single pore and multipore membranes submitted to other zero-average, periodically fluctuating potentials (square wave, sinusoidal, and triangular input signals) were also carried out, giving experimental efficiencies in the range $1-10 \%$. These values are relatively large because of the high amplitude of the input signals used. Maximum energy conversion efficiencies of the order of $1 \%$ [23] and up to $10 \%$ [24] have been theoretically predicted with electro-osmotic flow processes based on negatively charged conical nanopores.

Note that the above energy conversion processes are based on nanodevices integrated in small area membrane samples. In particular, practical energy harvesting aspects and long term storage have not been addressed. Simple order of magnitude estimations show that much higher membrane areas than that used here (less than $1 \mathrm{~cm}^{2}$ ) would be necessary for charging a small battery within reasonable times. Therefore, we expect our results to be useful only for small- 
scale energy transduction processes in nanopore-based sensors and bioelectronics interfaces immersed in electrolyte solutions.

\section{Conclusions}

We have shown theoretically and experimentally that significant energy conversion from external fluctuating signals (white noise) can be achieved using the electrical rectification of nanofluidic diodes. In particular, load capacitor voltages of the order of $1 \mathrm{~V}$ can be obtained in times of the order of $1 \mathrm{~min}$ with asymmetric single-nanopore and multipore membranes which convert zero time-average potentials of amplitudes of the order of $1 \mathrm{~V}$ into average net currents. We have used these membranes to demonstrate that the process is robust and can be enhanced significantly by simple scaling.

We have considered different electrolyte concentrations and operating conditions, addressing also the tuning of the load capacitance at each concentration (pore resistance). The experimental results suggest that soft matter nanostructures can be electrically coupled to conventional electronic elements, which is an essential characteristic for energy transduction in sensing nanopores and biological interfaces [4,12]. The experimental results can be described theoretically by using simple equivalent circuits with a voltage-dependent resistance in series with a load capacitance.

We note finally that the input signal amplitudes could be decreased to $0.1 \mathrm{~V}$ only for relatively thin, wide pore biological ion channels such as the OmpF porin of Escherichia coli bacteria [12] at the price of decreased physico-chemical robustness. Note also that these small pores should allow the use of external signals with higher frequencies than those employed here for our artificial biomimetic nanomaterials [21]. Additional work concerning this question is now in progress. 


\section{Acknowledgements}

We acknowledge the support from the Ministry of Economic Affairs and Competitiveness and FEDER (project MAT2012-32084) and the Generalitat Valenciana (project Prometeo/GV/0069).

\section{References}

[1] V. Gomez, P. Ramirez, J. Cervera, S. Nasir, M. Ali, W. Ensinger, S. Mafe, Charging a Capacitor from an External Fluctuating Potential using a Single Conical Nanopore, Sci. Rep. 5 (2015) 9501

[2] Z. Siwy, A. Fuliński, A nanodevice for rectification and pumping ions, Am. J. Phys. 72 (2004) 567.

[3] J. Cervera, P. Ramirez, S. Mafe, P. Stroeve, Asymmetric nanopore rectification for ion pumping, electrical power generation, and information processing applications, Electrochim. Acta 56 (2011) 4504.

[4] N. Misra, J. A. Martinez, S.-C. J. Huang, Y. Wang, P. Stroeve, C. P. Grigoropoulos, A. Noy, Bioelectronic silicon nanowire devices using functional membrane proteins, Proc. Natl. Acad. Sci. U.S.A. 106 (2009) 13780.

[5] W. Guan, S. Xin Li, M. A. Reed, Voltage gated ion and molecule transport in engineered nanochannels: theory, fabrication and applications, Nanotechnology 25 (2014) 122001.

[6] M. Tagliazucchi, I. Szleifer, Transport mechanisms in nanopores and nanochannels: can we mimic nature? Mater. Today 18 (2015) 131.

[7] Y. Hou, R. Vidu, P. Stroeve, Solar energy storage methods, Ind. Eng. Chem. Res. 50 (2011) 8954.

[8] W. Guo, L. Cao, J. Xia, F.-Q. Nie, W. Ma, J. Xue, Y. Song, D. Zhu, Y. Wang, L. Jiang, Energy harvesting with single-ion-selective nanopores: A concentration-gradient-driven nanofluidic power source, Adv. Funct. Mater. 20 (2010) 1339.

[9] P. Ramirez, M. Ali, W. Ensinger, S. Mafe, Information processing with a single multifunctional nanofluidic diode, Appl. Phys. Lett. 101 (2012) 133108.

[10] K. Tybrandt, R. Forchheimer, M. Berggren, Logic gates based on ion transistors. Nat. Commun. 3 (2012) 871.

[11] M. Ali, S. Nasir, P. Ramirez, J. Cervera, S. Mafe, W. Ensinger, CarbohydrateMediated Biomolecular Recognition and Gating of Synthetic Ion Channels, J. Phys. Chem. C 117 (2013) 18234. 
[12] M. Queralt-Martin, E. Garcia-Gimenez, V. M. Aguilella, P. Ramirez, S. Mafe, A. Alcaraz, Electrical pumping of potassium ions against an external concentration gradient in a biological ion channel, Appl. Phys. Lett. 103 (2013) 043707.

[13] A. Alcaraz, E. M. Nestorovich, M. L. López, E. García-Giménez, S. M. Bezrukov, V. M. Aguilella, Diffusion, exclusion, and specific binding in a large channel: a study of OmpF selectivity inversion, Biophys J. 96 (2009) 56.

[14] P. Apel, Track etching technique in membrane technology, Radiat. Meas. 34 (2001) 559.

[15] M. Ali, P. Ramirez, S. Mafe, R. Neumann, W. Ensinger, A pH-tunable nanofluidic diode with a broad range of rectifying properties. ACS Nano 3 (2009) 603.

[16] J. Cervera, B. Schiedt, R. Neumann, S. Mafe, P. Ramirez, Ionic conduction, rectification, and selectivity in single conical nanopores. J. Chem. Phys. 124 (2006) 104706.

[17] J. Cervera, P. Ramírez, J. A. Manzanares, S. Mafé, Incorporating ionic size in the transport equations through charged nanopores. Microfluid. Nanofluid. 9 (2010) 41.

[18] P. Ramirez, V. Gomez, M. Ali, W. Ensinger, S. Mafe, Net currents obtained from zero-average potentials in single amphoteric nanopores. Electrochem. Commun. 31 (2013)137.

[19] D. Momotenko, H. H. Girault, Scan-rate-dependent ion current rectification and rectification inversion in charged conical nanopores. J. Am. Chem. Soc. 133 (2011) 14496.

[20] V. Gomez, P. Ramirez, J. Cervera, S. Nasir, M. Ali, W. Ensinger, S. Mafe. Converting external potential fluctuations into nonzero time-average electric currents using a single nanopore, Appl. Phys. Lett.106 (2015) 073701.

[21] C. Verdia-Baguena, M. Queralt-Martin, V. M. Aguilella, A. Alcaraz, Protein Ion Channels as Molecular Ratchets. Switchable Current. Modulation in Outer Membrane Protein F Porin Induced by Millimolar La ${ }^{3+}$ Ions, J. Phys. Chem. C 116 (2012) 6537. 
[22] J. A. Quinn, J. L. Anderson, W. S. Ho, W. J. Petzny, Model pores of molecular dimension - Preparation and characterization of track-etched membranes, Biophys. J. 12 (1972) 990 .

[23] H.-C. Yeh, C.-C. Chang, R.-J. Yang, Electro-osmotic pumping and ionconcentration polarization based on conical nanopores, Phys. Rev. E, 91 (2005) 062302.

[24] C.-C. Chang, R.-J. Yang, Electrokinetic Energy Conversion Efficiency in Ionselective Nanopores, Appl. Phys. Lett., 99 (2011), 083102. 


\section{Figure captions}

\section{Fig. 1}

(a) Scheme of the experimental set-up. (b) the steady-state current $(I)$-voltage $\left(V_{N}\right)$ curves for three different samples of a membrane with a single conical pore. (c) the single pore electrical rectification as a function of $V_{N}$. (d) fluctuating electric potential $V(t)$ (white noise) of amplitude $V_{0}$ obtained from an external voltage source (input) and instantaneous electric current $I(t)$ (output) for sample 3.

\section{Fig. 2}

(a) The average current $\langle I\rangle$ as a function of the voltage amplitude $V_{0}$ for the three samples of Fig. 1. (b) the electrical resistance $R=V_{N} / I$ as a function of the voltage $V_{N}$. (c) the net current $<I>$ is used to charge the load capacitor in the circuit of Fig. 1 to voltages $V_{C}$ for $V_{0}=1 \mathrm{~V}$. (d) the capacitor discharging curves corresponding to the charging curves of (c). The load capacitance $C=1 \mu \mathrm{F}$ and the electrolyte concentration $c_{0}=0.1 \mathrm{M} \mathrm{KCl}$ in these experiments.

\section{Fig. 3}

(a) The steady-state $I-V_{N}$ curves of a multipore membrane. (b) the membrane electrical rectification as a function of $c_{0}$. (c) the electrical resistances $R$ as a function of $V_{N}$. (d) the capacitor charging curves for the white noise fluctuating signal. All experimental curves have been obtained at salt concentrations $c_{0}$ between 0.01 and $1 \mathrm{M} \mathrm{KCl}$.

\section{Fig. 4}

Experimental (points) and theoretical (continuous lines) capacitor charging (a) and discharging (b) curves for the multipore membrane and the load capacitances $C=1$ and $10 \mathrm{mF}$. The electrolyte concentration $c_{0}=0.1 \mathrm{M}$ and the voltage amplitude $V_{0}=2 \mathrm{~V}$.

\section{Fig. 5}


Experimental (points) and theoretical (continuous lines) capacitor charging (a) and discharging (b) curves obtained using the multipore membrane instead of the single pore membrane in Fig. 1a for different voltage amplitudes $V_{0}$. The load capacitance $C=10 \mathrm{mF}$ and the electrolyte concentration $c_{0}=0.1 \mathrm{M}$ in these experiments.

\section{Fig. 6}

The energy $E$ stored in the load capacitor as a function of the concentration $c_{0}$ at $V_{0}=2 \mathrm{~V}$ (a) and the voltage amplitude $V_{0}$ at $c_{0}=0.1 \mathrm{M}(\mathrm{b})$. In the two cases, $C=10 \mathrm{mF}$. 

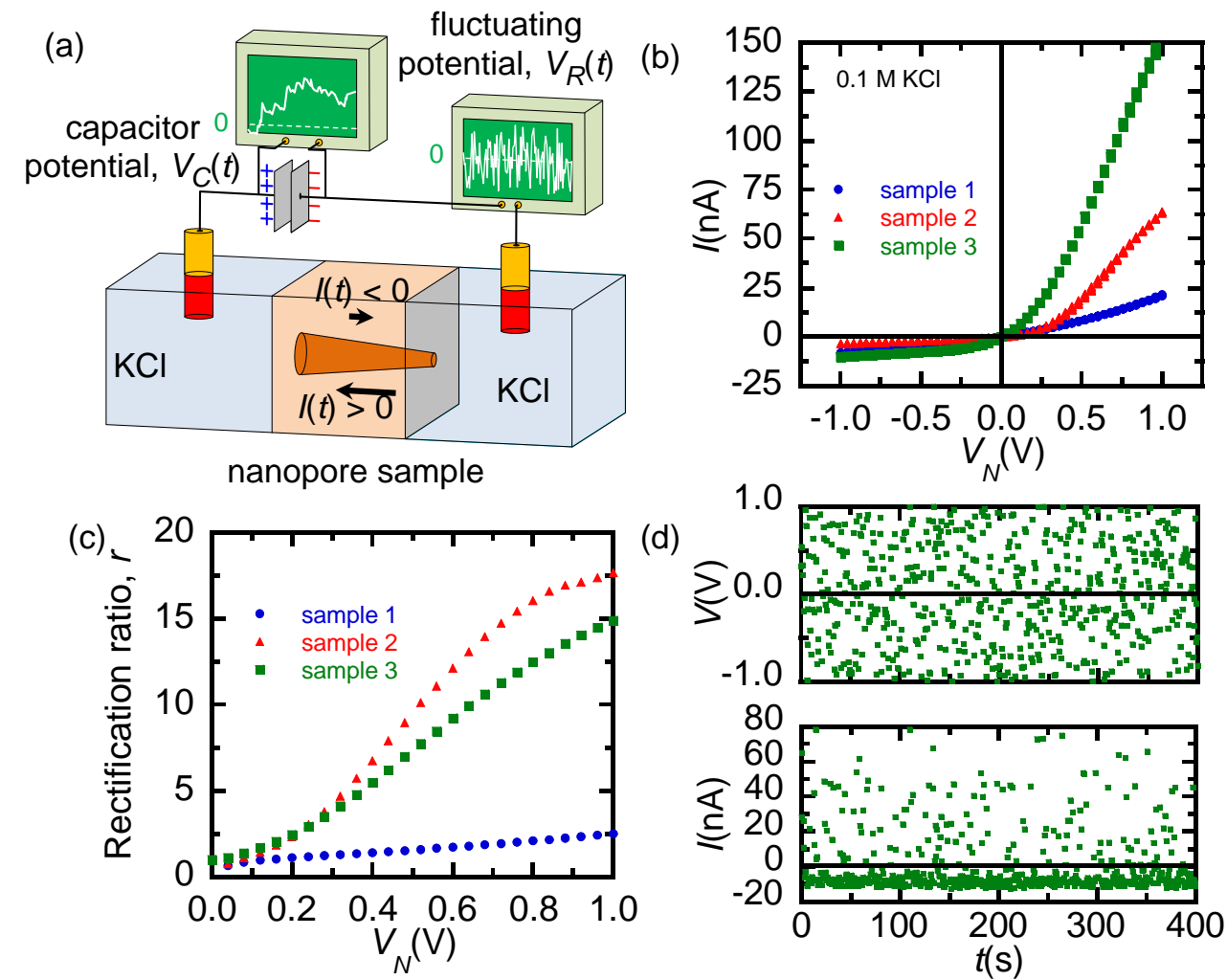

Fig. 1 

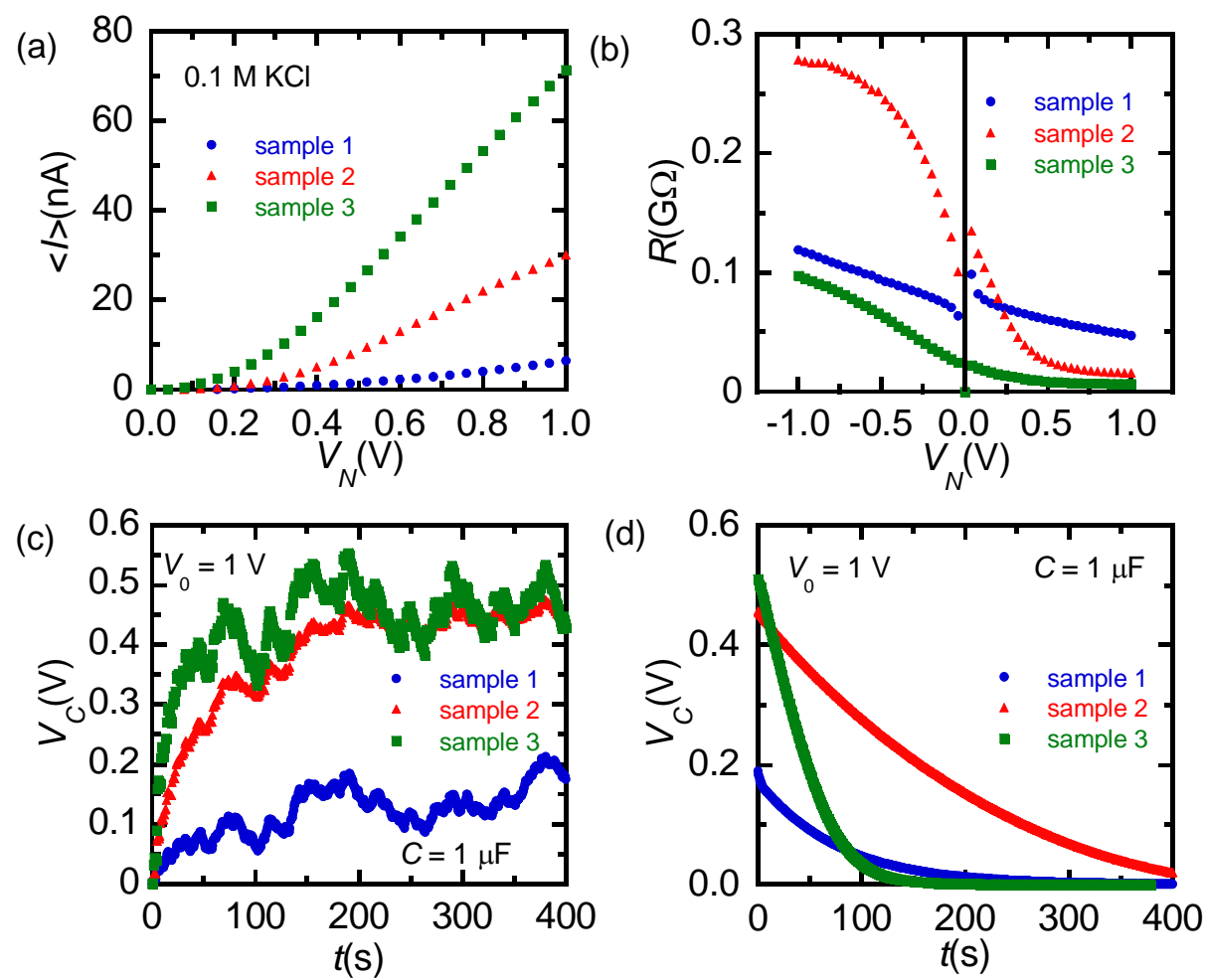

Fig. 2 

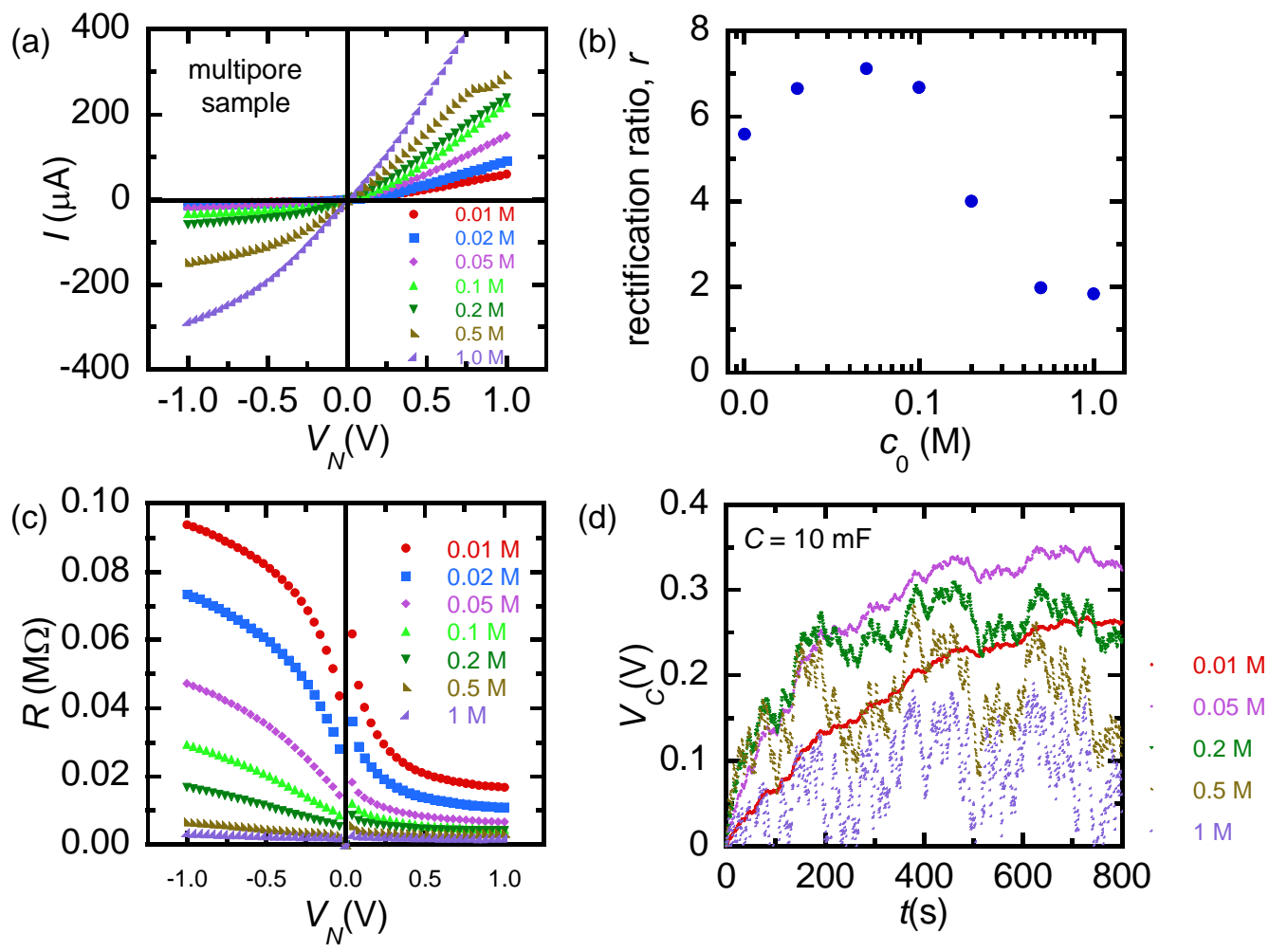

Fig. 3 

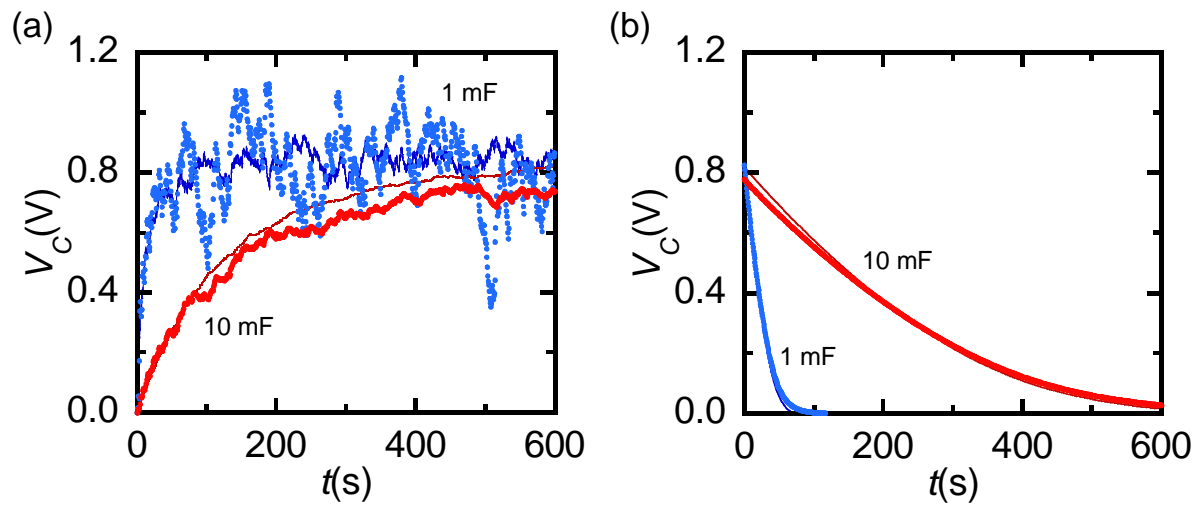

Fig. 4 

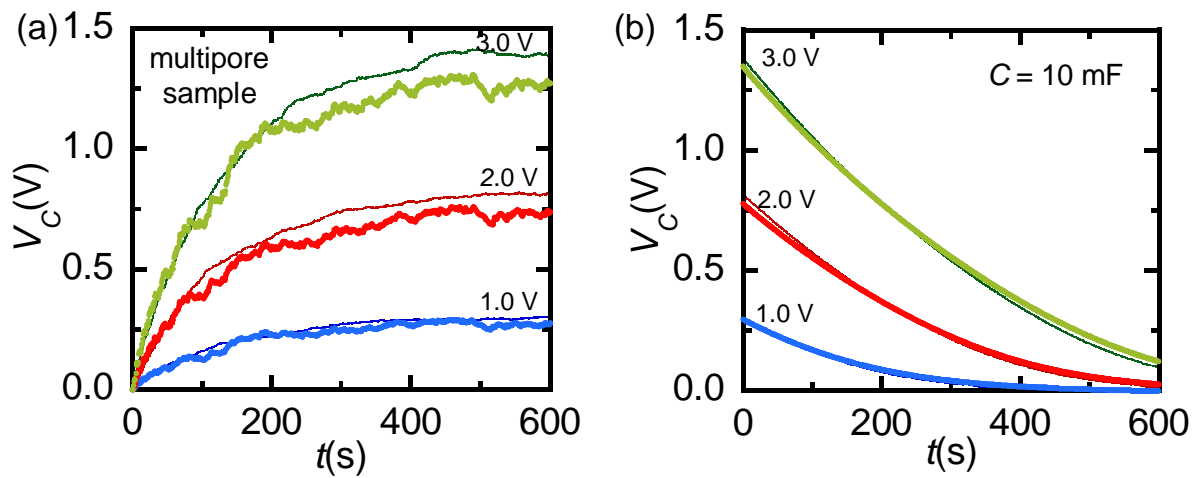

Fig. 5 

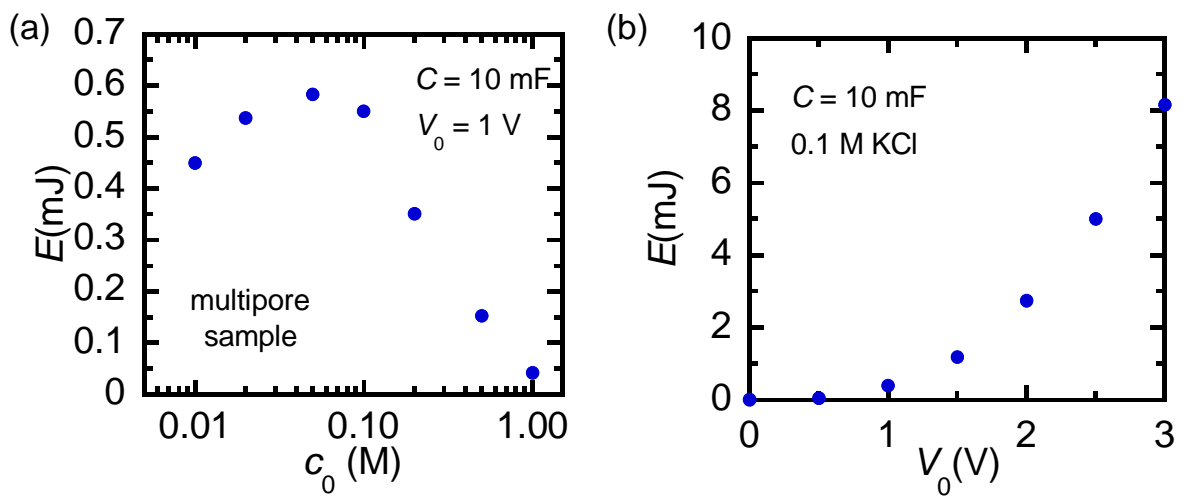

Fig. 6 